\title{
Minireview
}

\section{State-of-the-art management of nasopharyngeal carcinoma: current and future directions}

\author{
M Agulnik' and LL Siu*,I \\ 'Department of Medical Oncology and Hematology, Princess Margaret Hospital, University Health Network, 610 University Avenue, Toronto, Ontario, \\ Canada M5G 2 M9
}

Nasopharyngeal carcinoma (NPC) is a distinct type of head and neck cancer. Approximately $70 \%$ of patients with newly diagnosed NPC present with locally advanced disease. Phase III clinical trials support the addition of chemotherapy to radiotherapy for the initial treatment of these patients. Once metastatic disease develops, practices become varied. Further experience needs to be gained with both targeted therapies and immunotherapy to gauge whether they will improve treatment outcomes in NPC. British Journal of Cancer (2005) 92, 799-806. doi:I0.1038/sj.bjc.6602449 www.bjcancer.com

Published online I March 2005

(c) 2005 Cancer Research UK

Keywords: nasopharyngeal carcinoma; chemotherapy; radiotherapy; molecular targeted agents; immunotherapy

Nasopharyngeal carcinoma (NPC) is a distinct type of head and neck cancer that differs from other malignancies of the upper aerodigestive tract with respect to epidemiology, pathology, clinical presentation and responses to treatment. Outside of endemic areas such as Southeast Asia, this type of tumour is extremely rare with an incidence of less than $1 / 100000$ (Fandi et al, 1994). Patients with stage I and II disease have a high rate of cure with radiotherapy (RT) alone, while the prognosis for those with distant metastatic spread remains poor. Approximately $70 \%$ of patients with newly diagnosed NPC present with locally advanced, nonmetastatic stage III or IV disease. For this patient population, surgery has a limited role and RT delivered in combination with chemotherapy has become the standard of care. However, the timing, dosing, duration and optimal regimens of chemotherapy drugs to be combined with RT remain controversial. Several randomised trials have evaluated neoadjuvant, concurrent and adjuvant chemotherapy in different combinations with RT. This review focuses on the evidence to support treatment of locally advanced NPC, and provides an update of promising therapies that are on the horizon.

\section{THE ADDITION OF CHEMOTHERAPY TO RT FOR LOCALLY ADVANCED NPC}

A Medline and American Society of Clinical Oncology abstract search was conducted using the keywords 'nasopharyngeal cancer/ carcinoma' and 'radiotherapy/radiation therapy' and 'chemotherapy'. Articles and abstracts were eligible for review if patients were randomly assigned to receive chemotherapy and RT or RT alone. There are 11 published papers and four abstracts, which present the results of 13 randomised clinical trials comparing chemotherapy with RT to RT alone in patients with locally advanced NPC

*Correspondence: Dr LL Siu; E-mail: lillian.siu@uhn.on.ca

Received 8 November 2004; revised II January 2005; accepted II January 2005; published online I March 2005
(Tables 1 and 2). Only studies published in the English literature were included in this review.

\section{Neoadjuvant chemotherapy}

Four trials have assessed the role of neoadjuvant chemotherapy followed by RT vs RT alone (VUMCA 1996; Chua et al, 1998; Ma et al, 2001; Hareyama et al, 2002). The VUMCA I trial treated 339 patients in the neoadjuvant therapy arm with cisplatin, epirubicin and bleomycin. After a median follow-up duration of 49 months, this trial failed to show an overall survival (OS) advantage in the neoadjuvant therapy arm but did report a statistically significant increase in 3-year disease-free survival (DFS) rates (52 vs 32\%, interpolated). However, a treatment-related toxic death rate of $8 \%$ was encountered in the neoadjuvant therapy arm in this multicentre trial. In a small trial, Hareyama et al randomised 80 patients to two cycles of cisplatin and 5-fluorouracil administered prior to RT vs RT alone. Trends towards improved 5-year DFS and OS were demonstrated in the neoadjuvant therapy arm, but statistically significant differences were not achieved after a median follow-up period of 49 months. In their initial reporting after a median follow-up duration of 30 months, Chua et al showed similar results with their neoadjuvant combination of cisplatin and epirubicin. Again, trends towards improved OS and DFS were seen in the neoadjuvant therapy arm, but the results were not statistically significant. Lastly, Ma et al compared two to three cycles of bleomycin, cisplatin and 5-fluorouracil followed by RT to RT alone and showed a statistically significant prolongation of DFS in the chemotherapy group (59 vs $49 \%$ at 5 years). A trend towards OS was observed in the neoadjuvant arm of the trial. Updated combined data from these two latter trials (Chua DTT et al, 2004) on 784 patients, after a median follow-up period of 67 months, reported a statistically significant improvement in 5-year DFS (51 vs $43 \%$ ) favouring the neoadjuvant therapy arm, but not in 5-year OS (62 vs 58\%). Reductions in both locoregional and distant failures were observed. Although it has been suggested by the 
Table I Randomised trials of chemotherapy with RT vs RT alone in locally advanced NPC

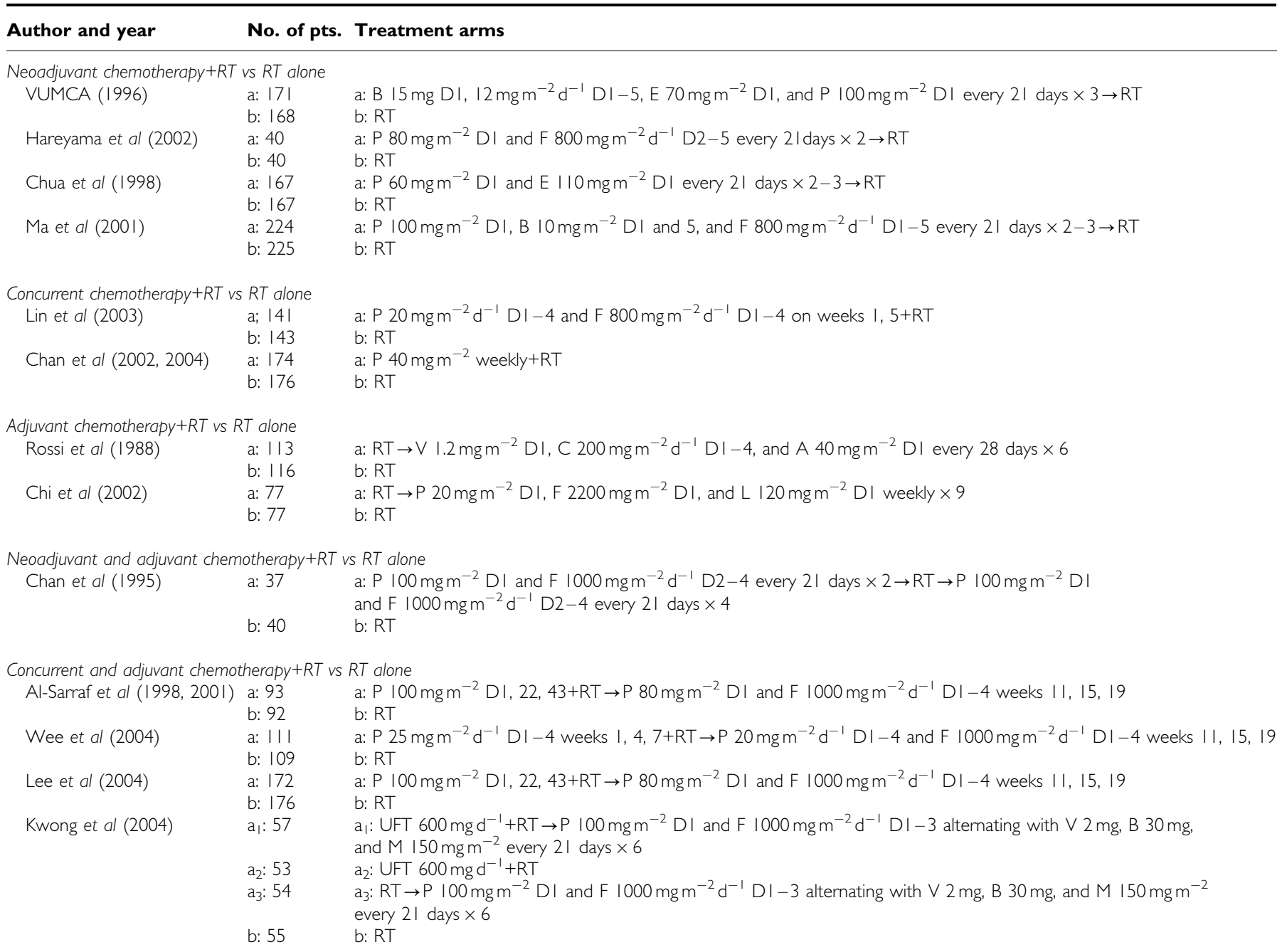

$\mathrm{NPC}=$ nasopharyngeal carcinoma; $\mathrm{RT}=$ radiotherapy; $\mathrm{P}=$ cisplatin; $\mathrm{F}=$ fluorouracil; $\mathrm{B}=$ bleomycin; $\mathrm{E}=$ epirubicin; $\mathrm{V}=$ vincristine; $\mathrm{C}=$ cyclophosphamide; $\mathrm{A}=$ adriamycin; $L=$ leucovorin; $M=$ methotrexate; UFT = uracil-tegafur; $a\left(a_{1}, a_{2}, a_{3}\right)=$ combined therapy arm; $b=$ radiotherapy alone arm; $d=$ day; $D=$ day; pts. = patients.

trends, to date, no statistically significant OS advantage has been documented in a phase III randomised trial using neoadjuvant chemotherapy followed by RT.

\section{Concurrent chemotherapy}

Two trials have compared concurrent chemotherapy and RT $v s$ RT alone (Chan et al, 2002; Lin et al, 2003). Lin et al randomised patients to concurrent chemoradiotherpy vs RT alone. Using the 1992 American Joint Committee on Cancer (AJCC) staging system, all 284 patients had either stage III or IV disease. Patients randomised to the concurrent chemotherapy arm of the study received two cycles of cisplatin mixed with 5-fluorouracil administered as a 96-h continuous infusion during weeks 1 and 5 of RT. The 5-year OS rates for the chemotherapy arm were $72 \%$ compared with $54 \%$ in the control arm; the 5-year DFS rates were 72 vs 53\%, respectively. Both comparisons were statistically significant. In a similar study, Chan et al randomised patients to adjuvant weekly low-dose cisplatin and standard RT vs RT alone. Over $90 \%$ of their patients were Ho tumour stage III or IV. The 2year DFS in the chemotherapy arm was $76 \%$ compared to $69 \%$ in the RT alone group, which did not achieve statistical significance, and OS was not reported in the initial analysis after a follow-up period of 33 months. Updated data from the Chan et al (2004) study, after a median follow-up duration of 65 months, reported 5year DFS of $60 v s 52 \%$ and 5-year OS of $70 v s 59 \%$, both reaching borderline statistical significance ( $P=0.06$ and 0.05 , respectively), in favour of the concurrent chemoradiotherapy arm. Reclassification of the 350 patients on this trial using the 1997 AJCC staging system revealed that over $70 \%$ were of stage III and IV. Subgroup analysis demonstrated that patients with $\mathrm{T} 3$ and $\mathrm{T} 4$ disease derived the most benefit. Based on these two randomised trials, patients with advanced locoregional NPC benefit from concurrent chemoradiotherapy over RT alone.

\section{Adjuvant chemotherapy}

The first published randomised trial in locoregional NPC compared adjuvant chemotherapy after RT to RT alone (Rossi et al, 1988). The regimen used in this 4-year multicentre randomised trial was vincristine, cyclophosphamide and adriamycin. Patients were treated with 6 monthly cycles of treatment following their RT. Analysis of the patient data at 4 years failed to show a statistically significant change in DFS or OS between the two groups. As well, interestingly, the pattern of failure in both groups was similar with distant metastasis accounting for $50 \%$ of 
Table 2 OS and DFS of randomised trials of chemotherapy with RT vs RT alone in locally advanced NPC

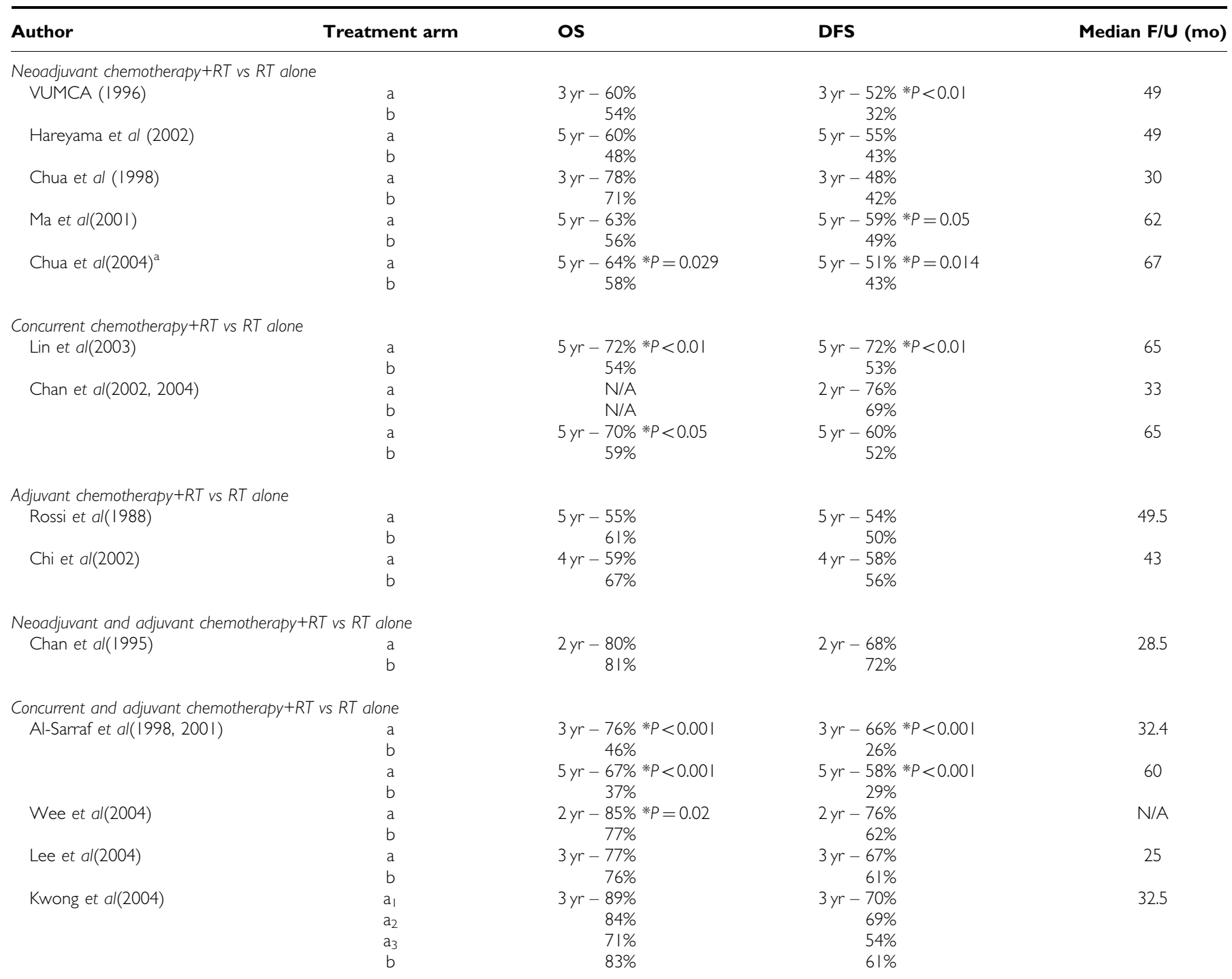

*Statistically significant result $(P \leqslant 0.05) . \quad a\left(a_{1}, a_{2}, a_{3}\right)=$ combined therapy arm; $b=$ radiotherapy alone arm; OS=overall survival; DFS=disease-free survival; $\mathrm{NPC}=$ nasopharyngeal carcinoma; $\mathrm{RT}=$ radiotherapy; $\mathrm{N} / \mathrm{A}=$ not applicable; $\mathrm{yr}=$ year; mo = months. ${ }^{\mathrm{a}}$ This reference is an updated analysis of patients from $\mathrm{Chua}$ et $a$ l (1998) and Ma et al (200I).

relapse failures. An important factor to consider is that the most active agent in NPC, cisplatin, has been omitted from their regimen. A second adjuvant trial did incorporate cisplatin with 5fluorouracil and leucovorin postradiation in patients with locally advanced NPC (Chi et al, 2002). The chemotherapy delivered in the adjuvant arm consisted of the three agents given as a 24 -h infusion, for 9 weekly cycles. At 5 years, there was no noted statistically different OS or DFS between the two groups of patients. Based on these two trials, adjuvant chemotherapy alone cannot be recommended for patients with advanced NPC.

\section{Neoadjuvant and adjuvant chemotherapy}

Only one phase III trial of 77 patients has evaluated the combined role of neoadjuvant and adjuvant chemotherapy (Chan et al, 1995). Neoadjuvant and adjuvant chemotherapy consisted of two and four cycles of cisplatin and 5-fluorouracil, respectively. After a follow-up duration of 28.5 months, no significant difference has been observed in either the DFS or OS.

\section{Concomitant and adjuvant chemotherapy}

A major breakthrough in the management of locally advanced NPC came about in 1998 with the publication of the phase III randomised Intergroup study 0099 (Al-Sarraf et al, 1998). Both arms of the study received identical RT, while the chemotherapy arm of the study received both concomitant and adjuvant chemotherapy. Cisplatin as a single agent was administered on days 1, 22 and 43 of the concurrent RT, and three adjuvant cycles of cisplatin with 5-fluorouracil were given monthly after completion of chemoradiotherapy. At 3 years, the DFS was $69 \%$ in the chemotherapy group and $24 \%$ in the RT alone arm. Their survival rates at 3 years were 78 vs 47\%, favouring chemotherapy. Updated analysis at 5 years (Al-Sarraf et al, 2001) confirms the benefit of treatment with 5-year DFS rates of $58 v s$ 29\% and 5-year OS rates of 67 vs 37\%, both favouring the combined therapy arm. The National Cancer database has been analysed for changing patterns of practice since the first published results of the Intergroup 0099 study data in 1998. Of all patients enrolled in the database and matching the eligibility criteria of the Intergroup 0099 study, only 
$38 \%$ received chemotherapy along with RT prior to 1997 , while since the publication of the data, $65 \%$ of these patients have received concurrent and adjuvant chemotherapy (Hoffman et al, 2004).

One-quarter of all patients treated on the Intergroup 0099 protocol had World Health Organization (WHO) stage I histology (keratinising squamous cell carcinoma). A phase III randomised trial using a similar chemotherapy and RT plan has been completed, with enrolment restricted to patients with WHO type IIa (nonkeratinising squamous cell carcinoma) and IIb (undifferentiated carcinoma) histologies (Wee et al, 2004). The chemotherapy regimen differed slightly from the Intergroup 0099 trial with the dose of cisplatin given in divided doses rather than one dose, but the total dose remained the same. The 2-year DFS and OS rates were statistically significant and favoured the use of chemotherapy. The Hong Kong NPC Study Group (Lee et al, 2004) randomised patients with nonkeratinising or undifferentiated NPC to the identical Intergroup 0099 regimen. On preliminary analysis after a median follow-up period of 2.3 years, the trends favoured the addition of chemotherapy with respect to DFS, but no benefit in OS was noted. Locoregional control was improved with the addition of chemotherapy.

In addition to the three trials mentioned above, which compared concurrent chemoradiotherapy plus adjuvant chemotherapy $v s \mathrm{RT}$ alone, a factorial study of four different regimens has been published (Kwong et al, 2004). This study assessed the combination of RT alone $v s$ three other schemas: RT with adjuvant chemotherapy, concurrent chemoradiotherapy and lastly, concurrent chemoradiotherapy followed by adjuvant chemotherapy. UFT (uracil and tegafur in 4:1 molar ratio) was used as the concurrent chemotherapy agent, while the adjuvant chemotherapy protocol consisted of alternating cycles of cisplatin/5-fluorouracil with vincristine/bleomycin/methotrexate. Although a trend towards improved DFS and OS was noted with the addition of concurrent chemotherapy, it did not reach statistical significance at 3 years. In assessing distant metastases rates, a significant reduction was attributable to concurrent chemotherapy. In this study, adjuvant chemotherapy did not improve outcome.

\section{SUMMARY OF EVIDENCE: THE ADDITION OF CHE- MOTHERAPY TO RT FOR LOCALLY ADVANCED NPC}

Based on published randomised trials that addressed the value of adding chemotherapy to RT in locally advanced NPC, several conclusions may be drawn. It is important to note that these trials were completed over a 16-year time span (1988-2004) in different geographic areas. As such, several tumour staging systems and improved diagnostic techniques have been utilised, which might have contributed to a phenomenon of stage migration (Feinstein et al, 1985). That withstanding, the timing of chemotherapy appears to impact on clinical outcome. Concurrent chemotherapy delivered with RT has consistently produced a survival benefit when compared to RT alone, achieving 5-year OS rates of about $70 \%$ with combined modality therapy in patients with nonmetastatic stage III and IV disease.

The addition of further chemotherapy to concurrent chemoradiotherapy, delivered in a neoadjuvant or adjuvant sequence, might further augment disease control. Reductions in both locoregional and distant failures have been reported with the addition of chemotherapy to RT, when delivered using validated regimens in adequate dosages. In reviewing the 13 randomised trials, chemotherapy compliance rates differed dramatically depending on the timing of therapy. In the neoadjuvant setting, $87-100 \%$ of patients received the prescribed cycles of chemotherapy, while $44-93 \%$ of patients scheduled for concurrent chemotherapy received their planned cycles. Even fewer, $14-55 \%$ of patients, completed their planned adjuvant chemotherapy. Clearly, chemotherapy dose intensity is most optimally maintained in the induction setting. This disparity in dose intensities may partially explain the lack of benefit associated with the administration of adjuvant chemotherapy alone. Treatment strategies to optimise chemotherapy delivery to achieve locoregional and distant control should utilise this information and consider the combination of induction chemotherapy followed by concurrent therapy. Incorporation of newer, less toxic and more effective anticancer agents such as gemcitabine, the taxanes or molecular targeted agents into combined modality regimens warrant continual exploration in locally advanced NPC. Collection of accurate toxicity data, especially those related to nutritional status, salivary and swallowing functions, ototoxicity, neuropathy and other late side effects, is vital to the planning of appropriate remedies.

\section{RT TECHNIQUES FOR LOCALLY ADVANCED NPC}

Tumour control of NPC has been highly correlated with the RT dose delivered to the tumour. In the above-mentioned randomised trials, RT dosing is summarised in Table 3. The dosing in all studies is similar and most are using a split field technique with two lateral opposed facial fields and an anterior field if necessary. A few studies enrolled patients and treated them with hyperfractionated RT twice per day, but the majority of patients in these studies still received conventional fractionation. Only one study included intracavitary brachytherapy for patients with persistent local disease (Chan et al, 2002).

In order to achieve tumour control for carcinoma of the nasopharynx, dosing of greater than 67 Gray (Gy) is required; however, better technical accuracy of RT delivery can also improve upon disease control. In 1998, the use of three-dimensional (3D) intensity-modulated RT (IMRT) for NPC was implemented at the Memorial Sloan-Kettering Cancer Centre. Initial treatment reported an increased target dose delivery from 67.0 to 77.3 Gy with IMRT as compared to conventional treatment methods. Dose to normal structures such as the spinal cord, mandible, temporal lobes and parotid glands all decreased with IMRT (Hunt et al, 2001). A 2-year follow-up of the initial 39 patients treated with IMRT confirms a local relapse-free survival (LRFS) of $97 v s 78 \%$ in historical controls treated with conventional technique with a 3D boost, suggesting an improved locoregional control with IMRT (Wolden et al, 2002).

The University of California - San Francisco has also extensively studied the use of IMRT in the treatment of NPC (Sultanem et al, 2000; Xia et al, 2000; Lee et al, 2002). Initially, IMRT treatment plans were compared with conventional treatment plans for a case of locally advanced NPC. Their planning confirmed that IMRT techniques improved tumour target coverage and spared sensitive normal tissue structures. Subsequently, 35 patients were treated with IMRT for NPC. In all, 72\% of these patients had 1997 AJCC stage III or IV disease. At 22 months of follow-up, no local or regional failures had been documented. This local control was attributable to improved tumour target coverage, increased total dose and increased dose per fraction to the tumour target. The mean dose delivered to the tumour target was $75.8 \mathrm{~Gy}$. Follow-up at 31 months with a total of 67 patients confirmed the above and reported only one local recurrence at the primary site. The vast majority of failures in this series were attributed to distant metastases. Of note, in this series, $75 \%$ of the patients did receive concurrent and adjuvant chemotherapy as per the Intergroup protocol 0099 regimen. The Prince of Wales Hospital (PWH) experience in Hong Kong further confirms the benefits of IMRT. In treating 63 newly diagnosed patients with NPC (stage I-IV disease), a 3-year LRFS of $92 \%$ and OS of $90 \%$ are observed (Kam et al, 2004).

Aside from documenting improved LRFS and OS with IMRT, both the UCSF and PWH experiences confirm favourable toxicity 
Table 3 Initial RT dose and schedule of randomised trials of chemotherapy with RT vs RT alone in locally advanced NPC

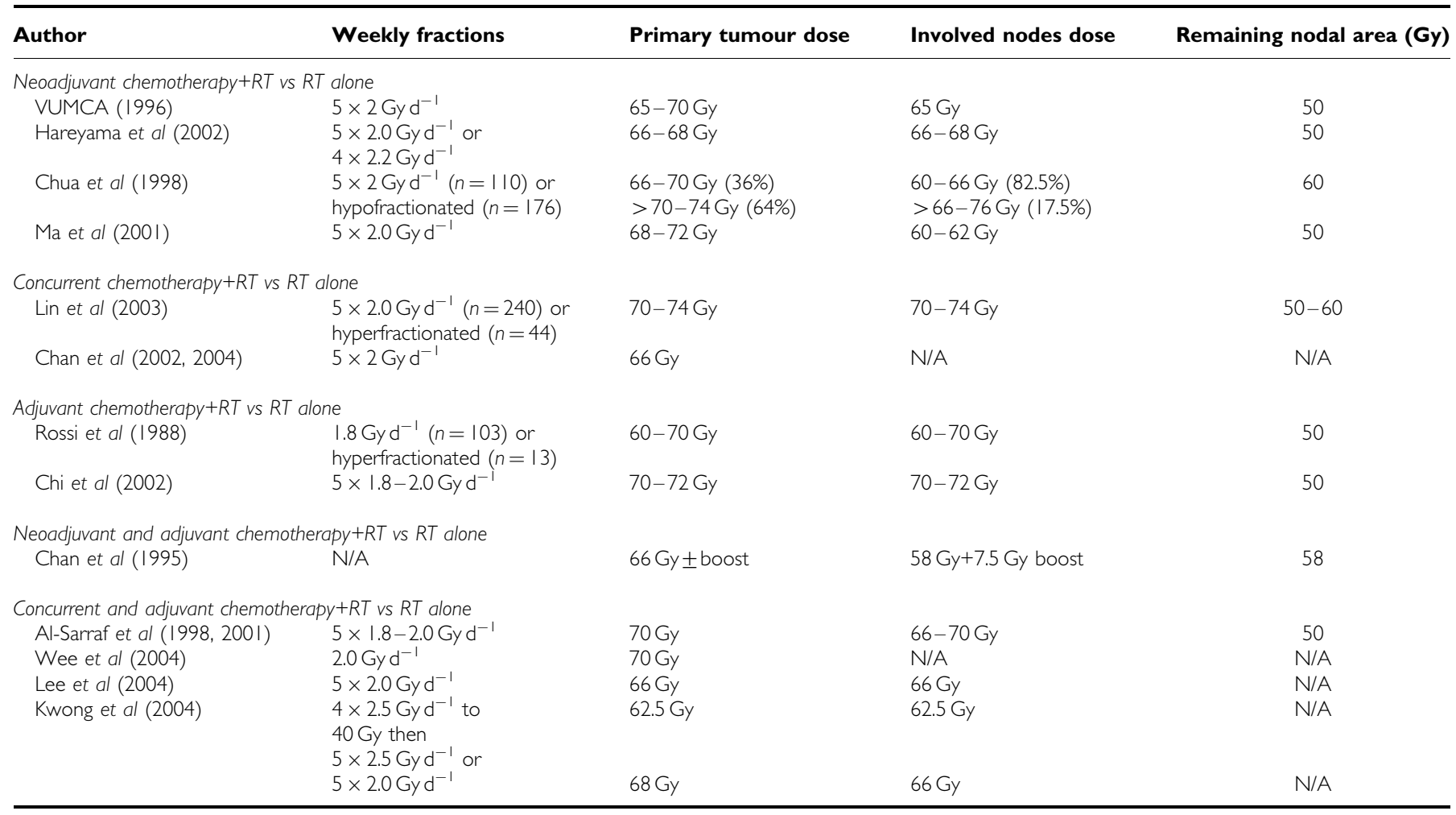

Gy = Grays; $d=$ day; N/A = not applicable; RT = radiotherapy; NPC = nasopharyngeal carcinoma; $a\left(a_{1}, a_{2}, a_{3}\right)=$ combined therapy arm, $b=$ radiotherapy alone arm.

profiles with IMRT. In spite of the increased dose, no increase in complication was reported, likely attributable to the reduced volumes to normal structures irradiated. Xerostomia is the most common side effect experienced by patients treated with conventional two-dimensional (2D) RT for NPC. Up to $97 \%$ of treated patients have reported xerostomia as a complication of therapy (Talmi et al, 2002). The UCSF data report no chronic xerostomia at 2 years, while the PWH experience reports $23 \%$ grade 2-3 xerostomia at 2 years with further decrease to $17 \%$ at 2 years in the subset of patients treated with less than $31 \mathrm{~Gy}$ to the parotid gland.

Although large randomised studies are not available to confirm the advantage of IMRT over conventional $2 \mathrm{D}$ and $3 \mathrm{D}$ RT techniques, the initial data are impressive in both locoregional disease control and reduced toxicities. A role for IMRT as a standard of care in the treatment of NPC is foreseeable. Aside from IMRT, other techniques are being explored in both early-stage and advanced NPC to improve upon locoregional response rates while limiting toxicities. Both high-dose-rate intracavitary brachytherapy and stereotactic radiosurgical boost have been shown to increase locoregional control rates when given as an adjuvant to external beam RT (Pai et al, 2002; Le et al, 2003; Lu et al, 2004). Given the preliminary data afforded by these phase II studies, the future direction of RT in the treatment of NPC will certainly be a move away from 2D RT to the adoption of 3D RT with IMRT, while a more defined role for stereotactic radio surgery and intracavitary brachytherapy in the management of locally advanced NPC still needs to be established.

\section{MOLECULAR TARGETED THERAPY}

Recurrent or metastatic NPC remains largely an incurable disease, although there have been reports of long-term survivors among those who achieved complete responses to conventional chemotherapy (Fandi et al, 2000). Combinations of cytotoxic chemotherapeutic agents with antitumour activity, such as the platinums, 5-fluorouracil, methotrexate, anthracyclines, gemcitabine and taxanes, typically yield high response rates of limited duration, and are associated with normal tissue toxicity. At the Princess Margaret Hospital, gemcitabine-based therapy has been adopted as first-line therapy for recurrent or metastatic NPC, with response rates of $34 \%$, as a single agent among platinum-refractory patients, or $64 \%$ when used in combination with cisplatin among platinum-sensitive patients. Median duration of response was 17 and 24 weeks, and 1-year survival rates were 48 and $69 \%$, respectively (Ma et al, 2002) In order to maximise the proportion of long-term survivors among patients with recurrent or metastatic NPC, better systemic agents are needed to increase the likelihood of complete responses. With a potentially superior therapeutic index, molecular targeted agents represent exciting compounds, which may complement the use of conventional chemotherapy in this disease.

Several molecular targets have been identified in tumour specimens of patient with NPC. Expression or overexpression of the following receptors has been evaluated in NPC: epidermal growth factor receptor (EGFR), vascular endothelial growth factor (VEGF), c-KIT and c-erbB-2 (HER2). To date, no reports of mutational analyses of any of these molecular targets have been published.

A retrospective study was performed to assess the correlation between the expression of EGFR and treatment outcome in patients with advanced stage disease (Chua DT et al, 2004). All patients in this group were treated with induction chemotherapy followed by RT. Of the specimens tested, $89 \%$ of the cases demonstrated EGFR expression. The extent and intensity of the staining did not correlate with tumour burden or extent of disease. 
Patients in whom greater than $25 \%$ of their tissue cells stained positive for EGFR had a reduced survival and increased risk of locoregional failure compared to those patients who did not. A second trial specifically evaluated undifferentiated carcinoma of the nasopharynx (Leong et al, 2004). EGFR expression levels were ascertained in 75 patients diagnosed with undifferentiated NPC. Rates of overexpression were reported at $83 \%$. In this cohort, the patients with EGFR overexpression did have a more locally aggressive disease, but DFS and OS rates did not alter significantly between the two groups. Given the finding of the high prevalence of EGFR overexpression, a phase II study of cetuximab (Erbitux, C-225) in combination with carboplatin for patients with metastatic or recurrent NPC was undertaken (Chan et al, 2003). Of 60 patients, 56 screened were positive for EGFR expression and enrolled in the trial. The overall response rate was $17 \%$, and partial response or stable disease accounted for $66 \%$. Based on this preliminary data, EGFR is a viable target for further clinical trials. Furthermore, a recent randomised phase III trial of RT with or without cetuximab in patients with locoregionally advanced non-nasopharyngeal squamous cell cancers of the head and neck has shown a significant survival benefit with the addition of cetuximab, and toxicity profile was acceptable. This trial has definite implications for the role of anti-EGFR agents in locally advanced NPC, and further evaluations are needed to determine how best to incorporate them into current standard chemoradiotherapy regimens (Bonner et al, 2004).

Vascular endothelial growth factor is an angiogenic factor, which contributes to angiogenesis and subsequent growth, invasion and metastasis of tumours. Expression of VEGF has been compared between tissue samples obtained from normal nasopharynx without tumour, nasopharyngeal benign tumours and NPC: the rates of VEGF expression were 10, 40 and $80 \%$, respectively. As well, expression of VEGF in advanced disease was increased with a statistical significance compared with those in early-stage disease (Guang-Wu et al, 2000). The role of anti-VEGF therapy has not been explored, but could serve as a potential target in future studies.

A retrospective study of 49 patients with NPC assessed the overexpression of HER2 and c-KIT (Bar-Sela et al, 2003). c-KIT overexpression was found in $33 \%$ of the cases and was associated with a positive Ebstein-Barr virus (EBV) status (by EBER in situ hybridisation) in patients whose histology showed nonkeratinising or undifferentiated carcinoma. These cases were associated with a trend towards a better survival. Whether or not a potential therapeutic role for inhibitors of c-KIT such as imatinib mesylate exists remains unknown at the present. In this same cohort of patients, HER2 overexpression was uniformly negative. This result differs from a Hong Kong study of 78 Chinese patients with undifferentiated NPC (Ma et al, 2003), in which HER2 overexpression was observed in $31 \%$ of the patients and conferred an association with increased stage of disease. Lastly, a study from Guangzhou in Southern China performed both immunohistochemical and fluorescent in situ hybridisation (FISH) analyses of HER2 in 45 cases of NPC. While $33 \%$ of patients had HER2 expression in tumour samples by immunohistochemical staining, this did not correlate with clinical outcome, and no significant alterations in gene copy number of HER2 was detected by FISH (Yan et al, 2002). At the present, no studies of trastuzumab or other HER2 inhibitors have been reported in NPC.

\section{EBV IN NPC AND IMMUNOTHERAPY}

A clear association exists between EBV infection and NPC. EBV is present in the cells of almost all primary and metastatic NPC, regardless of tumour histology, extent of disease or patient geographic location. It has been postulated that plasma EBV DNA quantification can be useful to follow patients and predict outcome of treatment (Lin et al, 2004). A recent study was conducted to investigate the significance of plasma EBV DNA concentrations in patients with advanced NPC. In all, 99 patients were treated with neodjuvant cisplatin and 5-fluorouracil given on alternate weeks for 10 weeks followed by RT therapy to a total of 70-74 Gy. Blood samples were obtained for measurement of EBV DNA concentrations using real-time quantitative polymerase chain reaction. Samples were obtained 1 day prior to beginning treatment, days 35 and 64 during neoadjuvant chemotherapy, and 1 week after completion of RT. Forty healthy volunteers, 20 patients cured of their disease for greater than 5 years and 19 patients with metastatic disease were used as controls. At baseline, 94 of 99 patients with stage III and IV disease, and all patients with metastatic disease had detectable plasma EBV DNA. No healthy controls had detectable EBV DNA. EBV DNA concentrations were significantly higher in the group that subsequently relapsed following therapy $v s$ the group that remained in a clinical remission. Higher pretreatment EBV DNA values corresponded to a decreased OS and DFS. Based on this initial data, EBV DNA quantification seems promising as a marker to monitor and predict treatment outcomes in patients with advanced NPC.

Given this association of EBV and NPC, investigators have evaluated a role for immunotherapy. In-depth studies have established that NPC cells express two distinct EBV latent membrane proteins, LMP-1 and LMP-2. These proteins become attractive targets for adoptive immunotherapy. A phase I study of EBV-specific cytotoxic T lymphocytes (CTL) was undertaken in EBV-positive patients with advanced NPC. CTL were generated and then infused into 10 patients. Four patients treated in remission remained disease free 19-27 months after infusion. Of six patients with refractory disease, two had complete responses, one had a partial response, one had stable disease and two had no response (Straathof et al, 2004). Except for the development of increased swelling at the site of pre-existing disease in one patient, CTL were generally well tolerated by all patients. Based on the preliminary work, the administration of CTL is feasible and is associated with significant antitumour activity. Further studies utilising CTL should be continued.

\section{CONCLUSIONS}

Based on the above data, a clear role for concomitant chemoradiotherapy followed by adjuvant chemotherapy has shown statistically significant improvement in OS and DFS for all histological types of locally advanced NPC. Neoadjuvant chemotherapy followed by concurrent chemoradiotherapy would be a reasonable variation, since the maintenance of chemotherapy dose intensity may be optimal using such a sequence. Further treatment questions arise in the management of local and distant failures. Intensity-modulated RT proves to be an improvement in RT delivery, and in several phase II studies has initially translated into better locoregional control and reduced toxicities. In cases of recurrent or distant disease, the standard of care is diverse.

Given the complex nature of this disease and the high risk for development of distant failures, new treatment regimens need to be developed for these patients. Exploration of the role of targeted agents such as inhibitors of EGFR, VEGF, c-KIT and HER2 are necessary. Adoptive immunotherapy with EBV-specific CTL awaits further exploration. Certainly, the treatment strategies for NPC will continue to change and evolve as a better understanding is gained of the molecular and immune mechanisms that drive this disease. 


\section{REFERENCES}

Al-Sarraf M, LeBlanc M, Giri PG, Fu KK, Cooper J, Vuong T, Forastiere AA, Adams G, Sakr WA, Schuller DE, Ensley JF (1998) Chemoradiotherapy $v s$ radiotherapy in patients with advanced nasopharyngeal cancer: phase III randomized Intergroup study 0099. J Clin Oncol 16: 1310-1317

Al-Sarraf M, LeBlanc M, Giri PGS, Fu K, Cooper J, Vuong T, Forastiere A, Adams G, Sakr W, Schuller D, Ensley J (2001) Superiority of five year survival with chemo-radiotherapy (CT-RT) vs radiotherapy in patients (pts) with locally advanced nasopharyngeal cancer (NPC). Intergroup (0099) (SWOG 8892, RTOG 8817, ECOG 2388) phase III study: final report. Proc Am Soc Clin Oncol 20: 227a (abstract 905)

Bar-Sela G, Kuten A, Ben-Eliezer S, Gov-Ari E, Ben-Izhak O (2003) Expression of HER2 and C-KIT in nasopharyngeal carcinoma: implications for a new therapeutic approach. Mod Pathol 16: 1035-1040

Bonner JA, Giralt J, Harari PM, Cohen R, Jones C, Sur RK, Rabin D, Azarnia $\mathrm{N}$, Needle MN, Ang KK (2004) Cetuximab prolongs survival in patients with locoregionally advanced squamous cell carcinoma of head and neck: a phase III study of high dose radiation therapy with or without cetuximab. J Clin Oncol 22: 489a (abstract 5507)

Chan AT, Ngan R, Teo P, Leung SF, Yiu HY, Yeo W, Mok T, Cheung FY, Kwan W, Zee B (2004) Final results of a phase III randomized study of concurrent weekly cisplatin-RT $v s$ RT alone in locoregionally advanced nasopharyngeal carcinoma (NPC). J Clin Oncol 22: 493a (abstract 5523)

Chan AT, Teo PM, Leung TW, Leung SF, Lee WY, Yeo W, Choi PH, Johnson PJ (1995) A prospective randomized study of chemotherapy adjunctive to definitive radiotherapy in advanced nasopharyngeal carcinoma. Int J Radiat Oncol Biol Phys 33: 569-577

Chan AT, Teo PM, Ngan RK, Leung TW, Lau WH, Zee B, Leung SF, Cheung FY, Yeo W, Yiu HH, Yu KH, Chiu KW, Chan DT, Mok T, Yuen KT, Mo F, Lai M, Kwan WH, Choi P, Johnson PJ (2002) Concurrent chemotherapyradiotherapy compared with radiotherapy alone in locoregionally advanced nasopharyngeal carcinoma: progression-free survival analysis of a phase III randomized trial. J Clin Oncol 20: 2038-2044

Chan ATC, Hsu MM, Goh BC, Hui EP, Liu TW, Millward M, Chang AY, Ma $\mathrm{BB}$, Hong RL, Lin X (2003) A phase II study of cetuximab (C225) in combination with carboplatin in patients (pts) with recurrent or metastatic nasopharyngeal carcinoma (NPC) who failed to a platinumbased chemotherapy. Proc Am Soc Clin Oncol 22: 497 (abstract 2000)

Chi KH, Chang YC, Guo WY, Leung MJ, Shiau CY, Chen SY, Wang LW, Lai YL, Hsu MM, Lian SL, Chang CH, Liu TW, Chin YH, Yen SH, Perng CH, Chen KY (2002) A phase III study of adjuvant chemotherapy in advanced nasopharyngeal carcinoma patients. Int J Radiat Oncol Biol Phys 52: $1238-1244$

Chua DT, Nicholls JM, Sham JS, Au GK (2004) Prognostic value of epidermal growth factor receptor expression in patients with advanced stage nasopharyngeal carcinoma treated with induction chemotherapy and radiotherapy. Int J Radiat Oncol Biol Phys 59: 11-20

Chua DT, Sham JS, Choy D, Lorvidhaya V, Sumitsawan Y, Thongprasert S, Vootiprux V, Cheirsilpa A, Azhar T, Reksodiputro AH (1998) Preliminary report of the Asian-Oceanian Clinical Oncology Association randomized trial comparing cisplatin and epirubicin followed by radiotherapy $v s$ radiotherapy alone in the treatment of patients with locoregionally advanced nasopharyngeal carcinoma. Asian-Oceanian Clinical Oncology Association Nasopharynx Cancer Study Group. Cancer 83: $2270-2283$

Chua DTT, Ma J, Sham JST (2004) Long-term survival after cisplatin-based induction chemotherapy and radiotherapy for nasopharyngeal carcinoma: a pooled data analysis of two phase III trials. J Clin Oncol 22: 494s (abstract 5524)

Fandi A, Altun M, Azli N, Armand JP, Cvitkovic E (1994) Nasopharyngeal cancer: epidemiology, staging, and treatment. Semin Oncol 21: $382-397$

Fandi A, Bachouchi M, Azli N, Taamma A, Boussen H, Wibault P, Eschwege F, Armand JP, Simon J, Cvitkovic E (2000) Long-term disease-free survivors in metastatic undifferentiated carcinoma of nasopharyngeal type. J Clin Oncol 18: $1324-1330$

Feinstein AR, Sosin DM, Wells CK (1985) The Will Rogers phenomenon. Stage migration and new diagnostic techniques as a source of misleading statistics for survival in cancer. $N$ Engl J Med 312: $1604-1608$

Guang-Wu H, Sunagawa M, Jie-En L, Shimada S, Gang Z, Tokeshi Y, Kosugi $\mathrm{T}$ (2000) The relationship between microvessel density, the expression of vascular endothelial growth factor (VEGF), and the extension of nasopharyngeal carcinoma. Laryngoscope 110: 2066-2069

Hareyama M, Sakata K, Shirato H, Nishioka T, Nishio M, Suzuki K, Saitoh A, Oouchi A, Fukuda S, Himi T (2002) A prospective, randomized trial comparing neoadjuvant chemotherapy with radiotherapy alone in patients with advanced nasopharyngeal carcinoma. Cancer 94: $2217-2223$

Hoffman H, Cooper JS, Weber R, Ang K, Porter K, Langer CJ (2004) Changing patterns of practice in the management of nasopharynx carcinoma (NPC): analysis of the National Cancer Database (NCDB). $J$ Clin Oncol 22: 488s (abstract 5501)

Hunt MA, Zelefsky MJ, Wolden S, Chui CS, LoSasso T, Rosenzweig K, Chong L, Spirou SV, Fromme L, Lumley M, Amols HA, Ling CC, Leibel SA (2001) Treatment planning and delivery of intensity-modulated radiation therapy for primary nasopharynx cancer. Int J Radiat Oncol Biol Phys 49: 623-632

Kam MK, Teo PM, Chau RM, Cheung KY, Choi PH, Kwan WH, Leung SF, Zee B, Chan AT (2004) Treatment of nasopharyngeal carcinoma with intensity-modulated radiotherapy: the Hong Kong experience. Int J Radiat Oncol Biol Phys 60: 1440-1450

Kwong DL, Sham JS, Au GK, Chua DT, Kwong PW, Cheng AC, Wu PM, Law MW, Kwok CC, Yau CC, Wan KY, Chan RT, Choy DD (2004) Concurrent and adjuvant chemotherapy for nasopharyngeal carcinoma: a factorial study. J Clin Oncol 22: $2643-2653$

Le QT, Tate D, Koong A, Gibbs IC, Chang SD, Adler JR, Pinto HA, Terris DJ, Fee WE, Goffinet DR (2003) Improved local control with stereotactic radiosurgical boost in patients with nasopharyngeal carcinoma. Int $J$ Radiat Oncol Biol Phys 56: 1046-1054

Lee AW, Lau WH, Tung SY, Chua D, Chappell R, Siu L, Chia S, Lau J, Law SCK (2004) Prospective randomized study on therapeutic gain achieved by addition of chemotherapy for T1-4N2-3M0 nasopharyngeal carcinoma (NPC). J Clin Oncol 22: 489s (abstract 5506)

Lee N, Xia P, Quivey JM, Sultanem K, Poon I, Akazawa C, Akazawa P, Weinberg V, Fu KK (2002) Intensity-modulated radiotherapy in the treatment of nasopharyngeal carcinoma: an update of the UCSF experience. Int J Radiat Oncol Biol Phys 53: 12-22

Leong JL, Loh KS, Putti TC, Goh BC, Tan LK (2004) Epidermal growth factor receptor in undifferentiated carcinoma of the nasopharynx. Laryngoscope 114: 153 - 157

Lin JC, Jan JS, Hsu CY, Liang WM, Jiang RS, Wang WY (2003) Phase III study of concurrent chemoradiotherapy $v s$ radiotherapy alone for advanced nasopharyngeal carcinoma: positive effect on overall and progression-free survival. J Clin Oncol 21: 631-637

Lin JC, Wang WY, Chen KY, Wei YH, Liang WM, Jan JS, Jiang RS (2004) Quantification of plasma Epstein-Barr virus DNA in patients with advanced nasopharyngeal carcinoma. $N$ Engl J Med 350: $2461-2470$

Lu JJ, Shakespeare TP, Tan LK, Goh BC, Cooper JS (2004) Adjuvant fractionated high-dose-rate intracavitary brachytherapy after external beam radiotherapy in $\mathrm{T} 1$ and $\mathrm{T} 2$ nasopharyngeal carcinoma. Head Neck 26: $389-395$

Ma BB, Poon TC, To KF, Zee B, Mo FK, Chan CM, Ho S, Teo PM, Johnson PJ, Chan AT (2003) Prognostic significance of tumor angiogenesis, Ki 67, p53 oncoprotein, epidermal growth factor receptor and HER2 receptor protein expression in undifferentiated nasopharyngeal carcinoma - a prospective study. Head Neck 25: $864-872$

Ma BB, Tannock IF, Pond GR, Edmonds MR, Siu LL (2002) Chemotherapy with gemcitabine-containing regimens for locally recurrent or metastatic nasopharyngeal carcinoma. Cancer 95: 2516-2523

Ma J, Mai HQ, Hong MH, Min HQ, Mao ZD, Cui NJ, Lu TX, Mo HY (2001) Results of a prospective randomized trial comparing neoadjuvant chemotherapy plus radiotherapy with radiotherapy alone in patients with locoregionally advanced nasopharyngeal carcinoma. J Clin Oncol 19: $1350-1357$

Pai PC, Chuang CC, Wei KC, Tsang NM, Tseng CK, Chang CN (2002) Stereotactic radiosurgery for locally recurrent nasopharyngeal carcinoma. Head Neck 24: 748-753

Rossi A, Molinari R, Boracchi P, Del Vecchio M, Marubini E, Nava M, Morandi L, Zucali R, Pilotti S, Grandi C, Ambrosini G, Cellini N, Chiavacci A, Colombo A, Dal Fior S, De Maria D, Felci U, Gabriele P, Laddaga M, Magno L, Marziano C, Olmi P, Prino A, Roncoroni L, Torretta A, Zampi G, Zorat PL, De Palo G (1988) Adjuvant chemotherapy with vincristine, cyclophosphamide, and doxorubicin after radiotherapy in local-regional nasopharyngeal cancer: results of a 4-year multicenter randomized study. J Clin Oncol 6: $1401-1410$

Straathof KC, Bollard CM, Popat U, Huls MH, Lopez T, Morriss MC, Gresik MV, Gee AP, Russell HV, Brenner MK, Rooney CM, Heslop HE (2004) 
Treatment of nasopharyngeal carcinoma with Epstein-Barr virusspecific T lymphocytes. Blood 2004 Nov 12 [Epub ahead of print]

Sultanem K, Shu HK, Xia P, Akazawa C, Quivey JM, Verhey LJ, Fu KK (2000) Three-dimensional intensity-modulated radiotherapy in the treatment of nasopharyngeal carcinoma: the University of California San Francisco experience. Int J Radiat Oncol Biol Phys 48: 711-722

Talmi YP, Horowitz Z, Bedrin L, Wolf M, Chaushu G, Kronenberg J, Pfeffer MR (2002) Quality of life of nasopharyngeal carcinoma patients. Cancer 94: $1012-1017$

VUMCA (1996) Preliminary results of a randomized trial comparing neoadjuvant chemotherapy (cisplatin, epirubicin, bleomycin) plus radiotherapy $v s$ radiotherapy alone in stage IV $(>$ or $=\mathrm{N} 2, \mathrm{M} 0)$ undifferentiated nasopharyngeal carcinoma: a positive effect on progression-free survival. International Nasopharynx Cancer Study Group. VUMCA I trial. Int J Radiat Oncol Biol Phys 35: 463-469
Wee J, Tan EH, Tai BC, Wong HB, Leong SS, Tan T, Chua ET, Lee KM, Yang E, Machin D (2004) Phase III randomized trial of radiotherapy $v s$ concurrent chemo-radiotherapy followed by adjuvant chemotherapy in patients with AJCC/UICC (1997) stage 3 and 4 nasopharyngeal cancer of the endemic variety. J Clin Oncol 22: 488s (abstract 5000)

Wolden S, Pfister D, Zelefsky M, Rosenzweig K, Chong L, Kraus D, Shah J, Leibel S (2002) Intensity modulated radiation therapy improves locoregional control for nasopharyngeal carcinoma. Proc Am Soc Clin Oncol 21: 240a (abstract 956)

Xia P, Fu KK, Wong GW, Akazawa C, Verhey LJ (2000) Comparison of treatment plans involving intensity-modulated radiotherapy for nasopharyngeal carcinoma. Int J Radiat Oncol Biol Phys 48: 329-337

Yan J, Fang Y, Huang BJ, Liang QW, Wu QL, Zeng YX (2002) Absence of evidence for HER2 amplification in nasopharyngeal carcinoma. Cancer Genet Cytogenet 132: 116-119 